\title{
ISOMORPHIC STEINER SYMMETRIZATION
}

\author{
B. KLARTAG and V.D. MILMAN
}

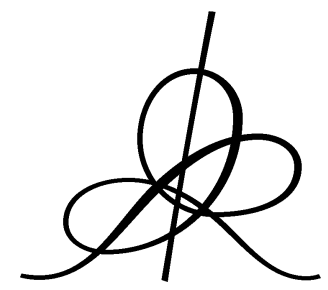

Institut des Hautes Études Scientifiques

35 , route de Chartres

91440 - Bures-sur-Yvette (France)

Septembre 2002

IHES/M/02/69 


\title{
Isomorphic Steiner Symmetrization
}

\author{
B. Klartag* V.D. Milman ${ }^{\dagger}$ \\ School of Mathematical Sciences, \\ Tel Aviv University, \\ Tel Aviv 69978, Israel
}

May 24, 2002

\begin{abstract}
This paper proves that there exist $3 n$ Steiner symmetrizations that transform any convex set $K \subset \mathbb{R}^{n}$ into an isomorphic Euclidean ball; i.e. if $\operatorname{vol}(K)=\operatorname{vol}\left(D_{n}\right)$ where $D_{n}$ is the standard Euclidean ball, then $K$ can be transformed into a body $\tilde{K}$ such that $c_{1} D_{n} \subset \tilde{K} \subset c_{2} D_{n}$, where $c_{1}, c_{2}$ are numerical constants. Moreover, for any $c>2$, cn symmetrizations are also enough.
\end{abstract}

\section{Introduction}

Fix a Euclidean structure (i.e. scalar product) in $\mathbb{R}^{n}$. Let $K \subset \mathbb{R}^{n}$ be any measurable set, and let $H=\left\{x \in \mathbb{R}^{n} ;\langle x, h\rangle=0\right\}$ be a hyperplane through the origin in $\mathbb{R}^{n}$. For every $x \in \mathbb{R}^{n}$ there exists a unique decomposition $x=y+t h$ where $y \in H, t \in \mathbb{R}$, so we can refer to $(y, t)$ as coordinates in $\mathbb{R}^{n}$. Define "Steiner symmetrization of $K$ with respect to $H^{\prime \prime}$ as the body:

$S_{H}(K)=\left\{(x, t) ; K \cap(x+\mathbb{R} h) \neq \emptyset,|t| \leq \frac{1}{2} \operatorname{Meas}\{K \cap(x+\mathbb{R} h)\}\right\}$

where Meas is the one dimensional Lebesgue measure in the line $x+$ $\mathbb{R} h$, normalized such that the measure of a segment is equal to its length. Steiner symmetrization preserves the $n$ dimensional volume of

\footnotetext{
*This work is part of a $\mathrm{PhD}$ thesis of the first named author, carried out under the supervision of the second named author, in Tel-Aviv university.

${ }^{\dagger}$ Supported by the Israel Science Foundation founded by the Academy of Sciences and Humanities, and by Minkowski center for Geometry.
} 
a set and transforms convex sets to convex sets (the so called "Brunn principle" [B1], [B2]).

Steiner symmetrizations were invented by Steiner [S] to prove the isoperimetric inequality. Throughout the last 160 years, Steiner symmetrizations have become a major tool for proving various geometric inequalities. Some samples are $[\mathrm{Mac}],[\mathrm{MeP}],[\mathrm{BZ}]$. It is clear that consecutive Steiner symmetrizations make the body closer to a Euclidean ball, in some sense. Indeed, in [CS], it is proved that for every $K$ there exists a sequence of symmetrizations of $K$ that converges, in the Hausdorff metric, to a Euclidean ball. In [Man] it is proved that even random symmetrizations are suitable, and convergence occurs with probability one. However, it was believed that many symmetrizations are needed for that convergence; An estimate of the order of $n^{n / 2}$ appears in $[\mathrm{H}]$.

Use of concentration phenomenon technique brought a tremendous improvement: a reduction to $c n \log n$ was achieved in [BLM]. More precisely, that paper proves:

Theorem 1.1 There exist universal constants $c, c_{1}, c_{2}>0$ such that for every convex body $K \subset \mathbb{R}^{n}$ with the same volume as the standard Euclidean ball, $D_{n}=\left\{x \in \mathbb{R}^{n} ;|x| \leq 1\right\}$, there exist cn $\log n$ Steiner symmetrizations that transform the body $K$ into $\tilde{K}$ such that:

$$
c_{1} D_{n} \subset \tilde{K} \subset c_{2} D_{n}
$$

In this paper we sharpen this estimate. Rather than $O(n \log n)$, we put $c n$ as the upper estimate for the number of required symmetrizations, where the constant "c" can be taken to be arbitrarily close to the value 2 . In addition, we prove that for every $\varepsilon>0$, only $\lfloor(1+\varepsilon) n\rfloor$ symmetrizations can transform an arbitrary body into an isomorphic ellipsoid. The latter estimate is asymptotically optimal. The following theorems are proved here:

Theorem 1.2 For every $\varepsilon>0$, there exist constants $c_{1}(\varepsilon), c_{2}(\varepsilon)>0$ such that for every convex body $K \subset \mathbb{R}^{n}$, there exist an ellipsoid $\mathcal{E}$ and $\lfloor(1+\varepsilon) n\rfloor$ Steiner symmetrizations that transform the body $K$ into $\tilde{K}$ such that:

$$
c_{1}(\varepsilon) \mathcal{E} \subset \tilde{K} \subset c_{2}(\varepsilon) \mathcal{E}
$$

Theorem 1.3 For every $\varepsilon>0$, there exist constants $c_{1}(\varepsilon), c_{2}(\varepsilon)>0$ such that for every convex body $K \subset \mathbb{R}^{n}$ with the same volume as the standard Euclidean ball, $D_{n}=\left\{x \in \mathbb{R}^{n} ;|x| \leq 1\right\}$, there exist $\lfloor(2+\varepsilon) n\rfloor$ Steiner symmetrizations that transform the body $K$ into $\tilde{K}$ such that:

$$
c_{1}(\varepsilon) D_{n} \subset \tilde{K} \subset c_{2}(\varepsilon) D_{n}
$$


We would like to point out that in addition to Theorem 1.2, for every convex body $K$ there exists a "position" - meaning a linear image of $K$ - such that regarding this image, $\lfloor(1+\varepsilon) n\rfloor$ symmetrizations are already enough to obtain an isomorphic Euclidean ball, for any $\varepsilon>0$. Hence, the larger amount of symmetrizations may be needed only due to a "wrong" position but not because of the geometry of the body itself.

Our results are of "isomorphic" nature, rather than "almost isometric". It is not proven that the symmetrizations make the body arbitrarily close to a Euclidean ball (compare with the situation in $[\mathrm{K}]$ ); We prove that the body becomes close to a Euclidean ball, up to some absolute constant, thus is "uniformly isomorphic" to a Euclidean ball. We are unaware of any good estimates regarding "almost-isometric" symmetrization (less than exponential in the dimension).

The paper is organized as follows: first, we describe a proof that the number of required symmetrizations is proportional to the dimension. This proof reduces the general problem to symmetrizing two specific bodies - the cube and the cross polytope. While the cross polytope is one of the most difficult bodies to symmetrize, the cube has a short symmetrization process, consisting only of $\lfloor\varepsilon n\rfloor$ symmetrizations. However, this method does not lead to the the best constants known to us. We then present proofs of the sharper results, using Milman's "quotient of subspace theorem". The reason for presenting the non-optimal proof, is that we think it is more accessible and may be interesting in itself. In addition, the proof that leads to the best constant is simplified when using the conclusion of the other proof as a lemma.

By $c, C, c^{\prime}$ etc. we denote universal constants, whose value may not be equal at different appearances in the paper. A "body" in $\mathbb{R}^{n}$ is any compact and convex set with non empty interior. The term "random" is used freely throughout the text, and we seldom bother to define the precise probability measure. The reason is that our standard manifolds (such as $S^{n}, O(n), G_{n, k}$ ) obey a canonical rotation invariant (Haar) probability measure, and all of our "random choices" are carried out with respect to these measures.

\section{Preliminary facts}

In this section we present some specific elementary properties of Steiner symmetrization, together with known facts to be used in the proof. 
Standard references for fundamental properties of Steiner symmetrization are the books [BZ], [BF]. See also [Sch] for general background on classical convexity theory.

Let $K \subset \mathbb{R}^{n}$ be a convex body and let $H=\left\{x \in \mathbb{R}^{n} ;\langle x, h\rangle=0\right\}$ be a hyperplane. Steiner symmetrization is denoted here by $S_{h}(K)$ or $S_{H}(K)$. When we say "Steiner symmetrization with respect to a vector", we mean with respect to the hyperplane orthogonal to that vector. Clearly,

$$
H \cap K \subset \operatorname{Proj}_{H}(K)=S_{H}(K) \cap H=\operatorname{Proj}_{H}\left(S_{H}(K)\right)
$$

where for a subspace $E$, we define $\operatorname{Proj}_{E}$ as the orthogonal projection onto $E$ in $\mathbb{R}^{n}$. Thus, if $E \subset H$ is a subspace, then $\operatorname{Proj}_{E}(K)=$ $\operatorname{Proj}_{E}\left(S_{h}(K)\right)$. Hence, by simple induction we proved:

Lemma 2.1 Let $H_{1}, . ., H_{k}$ be hyperplanes, and let $E \subset \bigcap_{i=1}^{k} H_{i}$ be a subspace. Denote $K^{\prime}=S_{H_{1}}\left(. . S_{H_{k}}(K) ..\right)$. Then:

$$
\begin{gathered}
\operatorname{Proj}_{E}(K)=\operatorname{Proj}_{E}\left(K^{\prime}\right) \\
E \cap K \subset E \cap K^{\prime}
\end{gathered}
$$

Useful corollary of Lemma 2.1: If $\operatorname{Proj}_{E}(K)=E \cap K$, and symmetrizations are carried out with respect to vectors in $E^{\perp}$, then both $E \cap K$ and $\operatorname{Proj}_{E}(K)$ remain unchanged.

Another elementary property of Steiner symmetrization is that it works independently in "fibers": The symmetrization acts independently in any affine translation of any subspace that contains $h$. Formally, as it follows from definitions,

Lemma 2.2 If $h \in F$ where $F$ is a subspace, then for every $x \in \mathbb{R}^{n}$,

$$
S_{h}(K) \cap(x+F)=S_{h}(K \cap(x+F))
$$

Therefore, if $\operatorname{Proj}_{E}(K)=E \cap K$, then any symmetrization with respect to any vector in $E$ would not change this. A second conclusion of this lemma is that if $K$ is centrally symmetric $(K=-K)$, then also $S_{h}(K)=-S_{h}(K)$. For a vector $x$ define $\pi_{x}$ as the reflection with respect to the hyperplane orthogonal to $x$. We say that $K$ is symmetric with respect to $x$ if $\pi_{x}(K)=K$. A third corollary of Lemma 2.2 is that if $K$ is symmetric with respect to $x$, symmetrizations with respect to vectors in $x^{\perp}$ would preserve this symmetry. In particular, 
Lemma 2.3 Let $K \subset \mathbb{R}^{n}$ be any set, and let $\left\{e_{1}, . ., e_{n}\right\}$ be an orthonormal basis. Then $K$ can be transformed to an unconditional set (i.e. symmetric with respect to the vectors $\left\{e_{i}\right\}$ ) using $n$ Steiner symmetrizations.

Generally, when we say that $K$ is an unconditional body, it should be understood that there exists an orthonormal basis, such that $K$ is symmetric with respect to its elements. The next claim is about orthogonal symmetrization. If $H_{1}, . ., H_{k}$ are orthogonal hyperplanes (i.e. their normals are mutually orthogonal), then the corresponding orthogonal projections $\operatorname{Proj}_{H_{1}}, . ., \operatorname{Proj}_{H_{k}}$ commute. Hence:

$$
S_{H_{1}, . ., H_{k}}(K) \supset \operatorname{Proj}_{H_{1} \cap . . \cap H_{k}}(K)
$$

where $S_{H_{1}, . ., H_{k}}(K)=S_{H_{1}}\left(. . S_{H_{k}}(K)\right.$..). Combining this with Lemma 2.1, we arrive at the following:

Lemma 2.4 Let $H_{1}, . ., H_{k}$ be orthogonal hyperplanes, and denote $E=$ $\bigcap_{i=1}^{k} H_{i}$ and $K^{\prime}=S_{H_{1}, . ., H_{k}}(K)$. Then:

$$
\operatorname{Proj}_{E}(K)=\operatorname{Proj}_{E}\left(K^{\prime}\right)=K^{\prime} \cap E
$$

Roughly speaking, Steiner symmetrizations can transform projections into sections. The next lemma is an addition to Lemma 2.4, and it sometimes represents the opposite idea (obtaining "good" projections, when the body already possesses "good" sections).

Lemma 2.5 Let $K \subset \mathbb{R}^{n}$ be a convex body which is centrally symmetric (i.e. $K=-K$ ), and let $H_{1}, . ., H_{k}$ be orthogonal hyperplanes, $E=\bigcap_{i=1}^{k} H_{i}$ and $K^{\prime}=S_{H_{1}, . ., H_{k}}(K)$, as before. Then:

$$
\operatorname{Proj}_{E}{ }^{\perp}\left(K^{\prime}\right)=K^{\prime} \cap E^{\perp}
$$

Proof: From the discussion following Lemma 2.2, $K^{\prime}$ is symmetric with respect to the vectors $h_{1}, . ., h_{k}$ (where $H_{i}=h_{i}^{\perp}$ ). If $x \in K^{\prime}$, then also $\pi_{h_{1} . .} \pi_{h_{k}}(x) \in K^{\prime}$. By convexity and central symmetry,

$$
\operatorname{Proj}_{E^{\perp}}(x)=\frac{x-\pi_{H_{1} . . \pi_{H_{k}}}(x)}{2} \in K^{\prime}
$$

hence $\operatorname{Proj}_{E}\left(K^{\prime}\right) \subset K^{\prime} \cap E^{\perp}$.

Lemma 2.6 Steiner symmetrization transforms an ellipsoid into an ellipsoid. In addition, for any ellipsoid $\mathcal{E} \subset \mathbb{R}^{n}$ there exists an orthonormal basis $\left\{v_{1}, . ., v_{n}\right\}$, such that symmetrizations of $\mathcal{E}$ with respect to the vectors $v_{1}, . ., v_{n}$ (in that order) transform it into a Euclidean ball. 
Proof: The proof is a small adjustment to the proof of Lemma 1.3 in [BLM]. By that lemma, ellipsoids are transformed to ellipsoids under Steiner symmetrization. We prove Lemma 2.6 by induction. Let $\mathcal{E}=\left\{x \in \mathbb{R}^{n} ; \sum_{i=1}^{n} x_{i}^{2} / a_{i}^{2} \leq 1\right\}$ be an ellipsoid. Let $r=\left(\prod a_{i}\right)^{1 / n}$. There exists a direction $u \in S^{n-1}$ such that $\mathcal{E} \cap \mathbb{R} u$ has length $2 r$. Let $\mathcal{E}_{1}$ be the Steiner symmetrization of $\mathcal{E}$ with respect to $u$. Then $\mathcal{E}_{1}$ is an ellipsoid, which has a prinicipal axis in the direction of $u$ of length $2 r$. Any symmetrizations with respect to vectors inside $u^{\perp}$ could not change this fact. By the induction hypothesis, the ellipsoid $\mathcal{E}_{1} \cap u^{\perp}$ can be symmetrized to a Euclidean ball of dimension $n-1$ and radius $r$, using symmetrizations with respect to an orthonormal basis of $u^{\perp}$. Apply exactly the same symmetrizations to the ellipsoid $\mathcal{E}_{1}$. The proof follows by Lemma 2.2 .

Before continuing, we would like to define two notions of distance between bodies in $\mathbb{R}^{n}$. For two sets $K, T \subset \mathbb{R}^{n}$ we denote their "geometric distance" as:

$$
d(K, T)=\inf \left\{a b ; \exists v \in \mathbb{R}^{n}, \frac{1}{b} K \subset T+v \subset a K\right\}
$$

The second notion of distance is the usual Bananch-Mazur distance (see e.g. [MS]) defined as:

$$
d_{B M}(K, T)=\inf \{d(u K, T) ; u \in G L(n)\}
$$

where $G L(n)$ is the set of invertible matrices acting on $\mathbb{R}^{n}$. Although the next theorem is not explicitly stated in [BLM], it can be easily deduced from their proof.

Theorem 2.7 There exist constants $\bar{c}_{1}, \bar{c}_{2}, \bar{c}>0$ such that for every convex body $K \subset \mathbb{R}^{n}$, there exist $\bar{c} n \log 2 d_{B M}\left(K, D_{n}\right)$ symmetrizations that transform $K$ into a body $\tilde{K}$ with:

$$
\bar{c}_{1} \text { v.r. }(K) D_{n} \subset \tilde{K} \subset \bar{c}_{2} \text { v.r. }(K) D_{n}
$$

where $D_{n}$ is the standard Euclidean ball in $\mathbb{R}^{n}$, and the volume-radius of $K$ is defined as v.r. $(K)=\left(\frac{\operatorname{vol}(K)}{\operatorname{vol}\left(D_{n}\right)}\right)^{\frac{1}{n}}$

\section{Isotropic Position}

In this section we use notions and properties related to isotropic positions. For a comprehensive discussion of this topic, see [MP1]. With any body $K, \operatorname{vol}(K)=1$, associate the Binet ellipsoid $\mathcal{B}(K)$, which is the unit ball of the Binet norm:

$$
\|x\|^{2}=\int_{K}\langle x, y\rangle^{2} d y
$$


If $\mathcal{B}(K)$ is homothetic to $D_{n}$, the standard Euclidean ball, we say that $K$ is in "isotropic position". Define for any $K$ (with $\operatorname{vol}(K)=1$ ) a number $L_{K}$ called "the isotropic constant of $K$ ":

$$
L_{K}^{2}=\frac{1}{n} \min _{\mathcal{E}} \int_{K}\|x\|_{\mathcal{E}}^{2} d x
$$

where the minimum runs over all ellipsoids with the same volume as $D_{n}$. If $K$ is in isotropic position, then the minimum is achieved for $\mathcal{E}=D_{n}$. It is computed in [MP1] that for a centrally symmetric $K$ :

$$
\left(\frac{\operatorname{vol}(\mathcal{B}(K))}{\operatorname{vol}\left(D_{n}\right)}\right)^{\frac{1}{n}}=\frac{1}{L_{K}}
$$

Lemma 3.1 The parameter $L_{K}$ decreases under Steiner symmetrization. If $K$ is a centrally symmetric body then $\operatorname{vol}(\mathcal{B}(K))$ increases.

Proof: By definition,

$$
L_{K}^{2}=\frac{1}{n} \min _{\mathcal{E}} \int_{K}\|x\|_{\mathcal{E}}^{2} d x
$$

where the minimum runs over all ellipsoids with the same volume as $D_{n}$. Let $\mathcal{E}$ be the ellipsoid where this minimum is achieved. For any affine line $l$ orthogonal to $H$, and for every $t>0$,

$$
\operatorname{Meas}(l \cap K \cap t \mathcal{E}) \leq \operatorname{Meas}\left(l \cap S_{H}(K) \cap S_{H}(t \mathcal{E})\right)
$$

and by Fubini,

$$
\int_{K}\|x\|_{\mathcal{E}}^{2} d x \geq \int_{S_{H}(K)}\|x\|_{S_{H}(\mathcal{E})}^{2} d x
$$

Since $S_{H}(\mathcal{E})$ has the same volume as $D_{n}$, it follows that $L_{S_{H}(K)} \leq L_{K}$. The other quantity in discussion is monotone in $L_{K}$.

For $\mathcal{E}$ a centrally symmetric ellipsoid and $H$ a hyperplane, denote by $S_{H}^{\circ}(\mathcal{E})$ the unique ellipsoid that satisfies three conditions:

(i) $\operatorname{Vol}\left(S_{H}^{\circ}(\mathcal{E})\right)=\operatorname{Vol}(\mathcal{E})$

(ii) $S_{H}^{\circ}(\mathcal{E}) \cap H=\mathcal{E} \cap H$

(iii) $S_{H}^{\circ}(\mathcal{E})$ is symmetric with respect to $H$.

It is easily verified that $\left(S_{H}\left(\mathcal{E}^{\circ}\right)\right)^{\circ}=S_{H}^{\circ}(\mathcal{E})$, where the polar (or dual) body of $K$ is defined as:

$$
K^{\circ}=\{x ; \forall y \in K\langle x, y\rangle \leq 1\}
$$


Lemma 3.2 Let $K$ be centrally symmetric body and $H$ a hyperplane. Then:

$$
S_{H}^{\circ}(\mathcal{B}(K)) \subset \mathcal{B}\left(S_{H}(K)\right)
$$

Proof: Let $x \in H$ be any vector. We claim that:

$$
\int_{K}|\langle x, y\rangle|^{2} d y=\int_{S_{H}(K)}|\langle x, y\rangle|^{2} d y
$$

Indeed, this follows directly by decomposing $\mathbb{R}^{n}=H \oplus H^{\perp}$, and using Fubini theorem. The Binet norms of $K$ and $S_{H}(K)$ are equal in the hyperplane $H$, therefore $\mathcal{B}\left(S_{H}(K)\right) \cap H=\mathcal{B}(K) \cap H$. In addition, $S_{H}(K)$ is symmetric with respect to $H$. Hence,

$$
\int_{S_{H}(K)}|\langle x, y\rangle|^{2} d y=\int_{S_{H}(K)}\left|\left\langle x, \pi_{H} y\right\rangle\right|^{2} d y=\int_{S_{H}(K)}\left|\left\langle\pi_{H} x, y\right\rangle\right|^{2} d y
$$

where $\pi_{H}$ is the reflection operator with respect to $H$. Thus the Binet ellipsoid of $S_{H}(K)$ is symmetric with respect to $H$. By Lemma $3.1, \mathcal{B}\left(S_{H}(K)\right)$ has at least the same volume as $\mathcal{B}(K)$. Both ellipsoids have the same intersection with $H$, the ellipsoid $\mathcal{B}\left(S_{H}(K)\right)$ is symmetric with respect to $H$, and has a greater (or equal) volume. By the definition of the operation $S_{H}^{\circ}$,

$$
S_{H}^{\circ}(\mathcal{B}(K)) \subset \mathcal{B}\left(S_{H}(K)\right)
$$

An important fact first observed by Bourgain [MP1], is that unconditional bodies satisfy $L_{K} \leq C$, with constant $C \leq \frac{1}{\sqrt{2}}$, as calculated in [BN]. We would like to give an ad-hoc definition of "almost isotropicity", to be used in the proof:

Definition 3.3 A body $K$ of volume one is called "almost isotropic with constant $C$ " if:

$$
C D_{n} \subset \mathcal{B}(K)
$$

Lemma 3.4 For any body $K \subset \mathbb{R}^{n}$ there exist $2 n$ Steiner symmetrizations that transform $K$ into a new body which is unconditional, and "almost isotropic with constant $\sqrt{2}$ ". The body will remain "almost isotropic with constant $\sqrt{2}$ " after any additional Steiner symmetrizations.

Proof: The first $n$ symmetrizations will be carried out with respect to any orthonormal basis. By Lemma 2.3 the body obtained $\tilde{K}$ is unconditional. Therefore $L_{\tilde{K}} \leq \frac{1}{\sqrt{2}}$. It is clear that $\tilde{K}$ is centrally 
symmetric. Combining (1) and the fact that for ellipsoids $v o l \mathcal{E} \cdot v_{o l} \mathcal{E}^{\circ}=$ $\left(\operatorname{vol}\left(D_{n}\right)\right)^{2}$ we get:

$$
\left(\frac{\operatorname{vol}\left(\mathcal{B}^{\circ}(K)\right)}{\operatorname{vol}\left(D_{n}\right)}\right)^{\frac{1}{n}} \leq \frac{1}{\sqrt{2}}
$$

where $\mathcal{B}^{\circ}(K)$ is the dual body to $\mathcal{B}(K)$. By Lemma 2.6, there exist $n$ vectors $v_{1}, . ., v_{n}$ such that

$$
S_{v_{1}}\left(S_{v_{2} \ldots} \ldots\left(\mathcal{B}^{\circ}(K)\right) \ldots\right)=t D_{n} \subset \frac{1}{\sqrt{2}} D_{n}
$$

Thus, by the definition of the operation $S^{\circ}$,

$$
\sqrt{2} D_{n} \subset S_{v_{1}}^{\circ}\left(S_{v_{2}}^{\circ} \ldots(\mathcal{B}(K)) \ldots\right)
$$

and any additional $S^{\circ}$ operations preserve (2). By Lemma 3.2, we get that for $K^{\prime}=S_{v_{1}}\left(S_{v_{2}} \ldots(K)\right.$..), it holds that

$$
\mathcal{B}\left(K^{\prime}\right) \supset \sqrt{2} D_{n}
$$

and that further Steiner symmetrizations of $K^{\prime}$ preserve the validity of (3). In addition, the body $K^{\prime}$ is unconditional, by Lemma 2.6 and Lemma 2.3 .

Remark for Lemma 3.4: Note that from the unproven "hyperplane conjecture" [MP1] it follows that just $n$ symmetrizations are enough for obtaining the conclusion of the lemma, since the first $n$ symmetrizations are unnecessary.

In a recent paper by Bobkov and Nazarov ([BN]), the following is proved:

Proposition 3.5 Let $\left\{e_{i}\right\}$ be an orthonormal basis in $\mathbb{R}^{n}$. Denote:

$$
B\left(l_{1}^{n}\right)=\left\{x \in \mathbb{R}^{n} ; \sum_{i}\left|\left\langle x, e_{i}\right\rangle\right| \leq 1\right\} \quad, \quad B\left(l_{\infty}^{n}\right)=\left\{x ; \forall i\left|\left\langle x, e_{i}\right\rangle\right| \leq 1\right\}
$$

the cross-polytope and the cube, respectively. Assume that $K$ is a body of volume one, which is unconditional with respect to $\left\{e_{i}\right\}$. Then:

$$
\max _{i} \int_{K}\left\langle x, e_{i}\right\rangle^{2} d x \leq C^{2} \Rightarrow \frac{C}{2} B\left(l_{\infty}^{n}\right) \subset K \subset \sqrt{3} C n B\left(l_{1}^{n}\right)
$$

It is clear that almost isotropic bodies satisfy the assumptions of Proposition 3.5 with $C \leq \frac{1}{\sqrt{2}}$. Hence, any unconditional "almost isotropic" body is bounded by a cube from below, and by a crosspolytope from above. Note that

$$
v \cdot r .\left(n B\left(l_{1}^{n}\right)\right)<c v . r .\left(B\left(l_{\infty}^{n}\right)\right)
$$


where $c \approx e$. Thus we should focus on symmetrizing the specific bodies $B\left(l_{1}^{n}\right)$ and $B\left(l_{\infty}^{n}\right)$ into isomorphic Euclidean balls. In the next sections we shall present two symmetrization procedures, for the cube and for the cross polytope. For every $0<\varepsilon<1$, we shall describe a symmetrization procedure of the cross-polytope consisting of $\lfloor(1+\varepsilon) n\rfloor$ symmetrizations, and a symmetrization procedure of the cube, that consists of $\lfloor\varepsilon n\rfloor$ symmetrizations.

To summarize the proposed symmetrization process of an arbitrary body $K \subset \mathbb{R}^{n}$ : Fix $0<\varepsilon<1$. Apply $2 n$ symmetrizations according to Lemma 3.4, to create unconditionality and almost-isotropicity. At this stage, by Proposition $3.5, K$ is contained inside an appropriate cross-polytope. Apply to $K$ the same $\lfloor(1+\varepsilon) n\rfloor$ symmetrizations that fit the cross-polytope. The symmetrizations are designed such that the resulting body would be unconditional again, hence will contain an appropriate cube. Now, apply the $\lfloor\varepsilon n\rfloor$ symmetrizations that are suitable for the cube. The resulting body $\hat{K}$ satisfies:

$$
c_{1}(\varepsilon) D_{n} \subset \frac{1}{v \cdot r .(K)} \hat{K} \subset c_{2} \varepsilon D_{n}
$$

where $c_{1}(\varepsilon), c_{2}(\varepsilon)>0$ are numerical constants, depending only on the parameter $\varepsilon$.

The total number of symmetrizations to be carried out is $\lfloor(3+$ $2 \varepsilon) n\rfloor$. This will prove that only $c n$ symmetrizations are needed in order to transform $K \subset \mathbb{R}^{n}$ to an isomorphic Euclidean ball (and any $c>3$ is adequate here).

\section{Steiner Symmetrizations of $B\left(l_{1}^{n}\right)$}

This section aims at symmetrizing a specific body: $K=\sqrt{n} B\left(l_{1}^{n}\right)$. Note that $D_{n} \subset K$. Since Steiner symmetrizations preserve $D_{n}$, it suffices to transform $K$, using Steiner symmetrizations, to a body $\tilde{K}$ such that:

$$
\tilde{K} \subset C D_{n}
$$

The general idea underlying the symmetrization process for $K$ is the fact discovered by Kashin [Ka]: the body $B\left(l_{1}^{n}\right)$ has very large sections which are almost Euclidean. Our symmetrization process consists of two stages. In the first stage, we symmetrize the body to create very large subspaces, such that the projections of the body to them are almost Euclidean. In the second stage, we bound Steiner symmetrizations by the Minkowski symmetrizations, and symmetrize in the small subspaces orthogonal to the large subspaces discussed earlier. 
Proposition 4.1 For any $\varepsilon>0$, there exist $\lfloor(1+\varepsilon) n\rfloor$ Steiner symmetrizations, that transform $K=\sqrt{n} B\left(l_{1}^{n}\right)$ into a body $\hat{K}$ such that:

$$
D_{n} \subset \hat{K} \subset c(\varepsilon) D_{n}
$$

Some lemmas are used in the proof. The first, which is a quantitative extension of Kashin's result, is due to Gluskin and Garnaev [GG]:

Lemma 4.2 Let $K=\sqrt{n} B\left(l_{1}^{n}\right)$, and let $E$ be a random subspace of dimension $\lambda n$. Then with high probability (greater than $1-e^{-c \lambda n}$ ),

$$
K \cap E \subset c_{1} \sqrt{\frac{-\log (1-\lambda)}{1-\lambda}} D_{n}
$$

For a body $K \subset \mathbb{R}^{n}$, define its supporting functional as $h_{K}(x)=$ $\sup _{y \in K}\langle x, y\rangle$. The mean width of $K$ is denoted as (see e.g. [MS])

$$
w(K)=2 M^{*}(K)=2 \int_{S^{n-1}} h_{K}(x) d \sigma(x)
$$

where $\sigma$ is the unique rotation invariant probability measure on the sphere.

Lemma 4.3 Let $K \subset \mathbb{R}^{n}$ be an unconditional body such that vol $(K)=$ $\operatorname{vol}\left(\sqrt{n} B\left(l_{1}^{n}\right)\right)$, and such that the body $\frac{1}{(\operatorname{vol}(K))^{1 / n}} K$ is "almost isotropic with constant $\sqrt{2} "$. Denote $\operatorname{diam}(K)=\sup _{x, y \in K}|x-y|$, the diameter of $K$. Then:

$$
M^{*}(K) \leq c_{2} \sqrt{\log (\operatorname{diam}(K))}
$$

Proof: By Bobkov-Nazarov (Proposition 3.5),

$$
K \subset c \sqrt{n} B\left(l_{1}^{n}\right)
$$

Since $K$ is unconditional, we have $K \subset \frac{\operatorname{diam}(K)}{2} D_{n}$, therefore $K \subset$ $c \sqrt{n} B\left(l_{1}^{n}\right) \cap \frac{\operatorname{diam}(K)}{2} D_{n}$. The result follows by calculating the mean width of the latter body (e.g. $[\mathrm{K}]$ ).

The next lemma is just a calculation (see e.g. [GM]):

Lemma 4.4 Let $K$ be a body in $\mathbb{R}^{n}, E$ is a subspace of dimension $\lambda n$. Then:

$$
M_{E}^{*}(K \cap E) \leq \frac{c_{3}}{\sqrt{\lambda}} M_{\mathbb{R}^{n}}^{*}(K)
$$


For a hyperplane $H$ define $\tau_{H}(K)=\frac{K+\pi_{H}(K)}{2}$, the Minkowski symmetrization $\left(\pi_{H}\right.$ is the reflection with respect to $\left.H\right)$. It is clear that $S_{H}(K) \subset \tau_{H}(K)$ (e.g. see [BLM]). Minkowski symmetrizations preserve $M^{*}(K)$, and in $[\mathrm{K}]$ it is proved that for every body, there exist $5 n$ Minkowski symmetrizations that transform the body into an approximate Euclidean ball, of radius $M^{*}(K)$. Hence,

Proposition 4.5 For every body $K \subset \mathbb{R}^{n}$, there exist $5 n$ Steiner symmetrizations such that $K$ is transformed into a body $\tilde{K}$ such that:

$$
\tilde{K} \subset c_{4} M^{*}(K) D_{n}
$$

and $\tilde{K}$ is an unconditional body, since the last $n$ symmetrizations are carried out with respect to an orthonormal basis.

\subsection{Initial symmetrizations}

Choose by random an orthonormal basis $\left\{e_{1}, . ., e_{n}\right\} \in O(n)$, and symmetrize $K$ with respect to $e_{n}, . ., e_{1}$ (in this order) to obtain $K^{\prime}$. Then clearly $K^{\prime}$ is unconditional with respect to $e_{1}, . ., e_{n}$.

Claim 4.6 With high probability of choosing the basis $\left\{e_{1}, . ., e_{n}\right\}$, for $1 \leq k \leq n$ :

$$
\operatorname{Proj}_{F_{k}} K^{\prime} \subset c_{1} \sqrt{\frac{n}{k} \log \frac{n}{k}} D_{n}
$$

where $F_{k}=s p\left\{e_{k+1}, . ., e_{n}\right\}$ and further symmetrizations with respect to vectors inside $s p\left\{e_{1}, . ., e_{k}\right\}$ cannot ruin the validity of (4).

Proof: Since the orthonormal basis $\left\{e_{1}, . ., e_{n}\right\}$ is chosen by random, then the subspaces $F_{k}=s p\left\{e_{k+1}, . ., e_{n}\right\}$ are random. According to Lemma 4.2

$$
K \cap F_{k} \subset c \sqrt{\frac{n}{k} \log \frac{n}{k}} D_{n} \cap F_{k}
$$

Now, the first $n-k$ symmetrizations cannot affect the validity of (5), since they preserve $D_{n} \cap F_{k}$, according to Lemma 2.2. Let $K^{\prime \prime}$ be the body obtained after the first $n-k$ symmetrizations. Then by Lemma 2.5 (the body is clearly centrally symmetric),

$$
\operatorname{Proj}_{F_{k}}\left(K^{\prime \prime}\right)=K^{\prime \prime} \cap F_{k} \subset c \sqrt{\frac{n}{k} \log \frac{n}{k}} D_{n} \cap F_{k}
$$

The next $k$ symmetrizations are carried out with respect to vectors orthogonal to $F_{k}$. Thus, by the corollary of Lemma 2.1, these $k$ symmetrizations cannot ruin (4). Furthermore, any additional symmetrization with respect to vectors in $s p\left\{e_{1}, . ., e_{k}\right\}$ cannot hurt the validity of (4). 


\subsection{Iteration of symmetrizations}

So far, we have performed $n$ Steiner symmetrizations to $K=\sqrt{n} B\left(l_{1}^{n}\right)$, and have obtained a body $K^{\prime}$ that satisfies Claim 4.6. We plan to perform a short series of $\lfloor\varepsilon n\rfloor$ iterations. Each iteration reduces both the diameter and the mean width at least logarithmically, and keeps the body unconditional for the next iteration. The $i^{t h}$ iteration would consist of $5\left\lfloor\frac{n}{\log ^{(i)} n}\right\rfloor$ Steiner symmetrizations (where $\log ^{(0)} n=n$, and $\left.\log ^{(i+1)} n=\log \log { }^{(i)} n\right)$. When we arrive at the first $i$ such that $\log ^{(i)} n<c_{6}(\varepsilon)=\max \left\{\log 2 c_{5}, 10 / \varepsilon\right\}$ we will stop, where $c_{5}=$ $\max \left\{2 c_{1}, 4 c_{2} c_{3} c_{4}\right\}$, a numerical constant. Thus, the entire process (including the initial symmetrizations) consists of

$$
n+\sum_{i} 5\left\lfloor\frac{n}{\log ^{(i)} n}\right\rfloor \leq\lfloor(1+\varepsilon) n\rfloor
$$

symmetrizations. Denote $E_{i}=s p\left\{e_{1}, . ., e_{\left\lfloor\frac{n}{\left.\log ^{(i)}\right)_{n}}\right\rfloor}\right\}$, and $K_{i}$ is the body obtained from the $i^{t h}$ iteration $\left(K_{0}=K^{\prime}\right)$. The definition of the $i^{t h}$ iteration is simple: Use $K_{i-1} \cap E_{i}$ as the object for Proposition 4.5. To arrive at $K_{i}$, apply the same set of symmetrizations (with respect to the same vectors in $E_{i}$ ) to the entire body $K_{i-1}$.

Claim 4.7 For $i$ such that $\log ^{(i)} n \geq c_{6}(\varepsilon)$, the body $K_{i}$ is unconditional and satisfies:

$$
\operatorname{diam}\left(K_{i}\right)<2 c_{5} \log ^{(i)} n
$$

Proof: By induction. For $i=0$ it is clear. Assume that

$$
\operatorname{diam}\left(K_{i-1}\right)<2 c_{5} \log ^{(i-1)} n
$$

and $K_{i-1}$ is unconditional. Since Steiner symmetrizations preserve "almost isotropicity", by Lemma 4.3 we have

$$
M^{*}\left(K_{i-1}\right) \leq c_{2} \sqrt{\log ^{(i)} n+\log 2 c_{5}}<2 c_{2} \sqrt{\log ^{(i)} n}
$$

where the latter inequality follows from definition of $c_{6}(\varepsilon)$. By Lemma 4.4, the mean width of $K_{i-1} \cap E_{i}$ with respect to its ambient subspace is bounded:

$$
M_{E_{i}}^{*}\left(K_{i-1} \cap E_{i}\right) \leq c_{3} \sqrt{\log ^{(i)} n}\left(2 c_{2} \sqrt{\log ^{(i)} n}\right)=2 c_{2} c_{3} \log ^{(i)} n
$$

Recall the definition of the iteration: $K_{i}$ was obtained from $K_{i-1}$ using symmetrizations in the subspace $E_{i}$, which were chosen according to Proposition 4.5. By Lemma 2.2,

$$
\operatorname{diam}\left(K_{i} \cap E_{i}\right)<2 c_{4} \cdot\left(2 c_{2} c_{3} \log ^{(i)} n\right)
$$


and by Lemma 2.5 , since $K_{i}$ is symmetric with respect to an orthonormal basis of $E_{i}$,

$$
\operatorname{diam}\left(\operatorname{Proj}_{E_{i}}\left(K_{i}\right)\right)=\operatorname{diam}\left(K_{i} \cap E_{i}\right)<2 c_{4} \cdot\left(2 c_{2} c_{3} \log ^{(i)} n\right)
$$

This is all regarding the subspace $E_{i}$. Take a look at its complement $E_{i}^{\perp}$. Note that all of the symmetrizations in the previous iterations were carried out inside $E_{i}$. By a corollary to Lemma $2.2 K_{i}$ is still symmetric with respect to an orthonormal basis in $E_{i}^{\perp}$, hence in total $K_{i}$ unconditional. In addition, by Claim 4.6, we still have:

$$
\operatorname{diam}\left(\operatorname{Proj}_{E_{i}^{\perp}}\left(K_{i}\right)\right)<2 c_{1} \sqrt{\log ^{(i)} n \log ^{(i+1)} n}<2 c_{1} \log ^{(i)} n
$$

Note that

$$
\operatorname{diam}\left(K_{i}\right) \leq \sqrt{2} \max \left\{\operatorname{diam}\left(\operatorname{Proj}_{E_{i}}\left(K_{i}\right)\right), \operatorname{diam}\left(\operatorname{Proj}_{E_{i}^{\perp}}\left(K_{i}\right)\right)\right\}
$$

and therefore $\operatorname{diam}\left(K_{i}\right)<\sqrt{2} c_{5} \log ^{(i)} n$

Hence, we have used $\lfloor(1+\varepsilon) n\rfloor$ symmetrizations for the body $K=\sqrt{n} B\left(l_{1}^{n}\right)$, and have obtained a body with diameter smaller than $2 c_{5} \log ^{(i-1)} n \leq 2 c_{5} e^{c_{6}(\varepsilon)}$. Thus, Proposition 4.1 is proved. In addition, it is clear that those $\lfloor(1+\varepsilon) n\rfloor$ symmetrizations, applied to any $K \subset \mathbb{R}^{n}$, will transform it into an unconditional body.

Remark for Proposition 4.1: The proven dependence in $\varepsilon$ here is exponential. However, the real dependence in $\varepsilon$ in the proposition is only $O\left(\sqrt{\frac{1}{\varepsilon} \log \frac{1}{\varepsilon}}\right)$. This can be proven in an analogous way to the discussion at the end of section 5 (and using Santalo inequality, for obtaining the dual form of Lemma 5.3).

\section{Steiner Symmetrizations of the cube}

Let $K=\frac{1}{\sqrt{n}} B\left(l_{\infty}^{n}\right)$. This section aims at proving the following:

Proposition 5.1 For any $0<\varepsilon<1$, there exist $\lfloor\varepsilon n\rfloor$ Steiner symmetrizations, that transform $K$ into a body $\hat{K}$ such that:

$$
c(\varepsilon) D_{n} \subset \hat{K} \subset D_{n}
$$

where $c(\varepsilon)$ is of the order of $O\left(\sqrt{\frac{\varepsilon}{-\log \varepsilon}}\right)$.

The proof given in this section resembles the proof in the preceding section, partially because $K$ is the dual body of $\sqrt{n} B\left(l_{1}^{n}\right)$ (for information about duality, see [MS] or [GM]). Note $K \subset D_{n}$, and therefore our concern is only about symmetrizing from below. In a dual manner 
to $B\left(l_{1}^{n}\right)$, our body has very large almost Euclidean projections. Recall that the purpose of the first $n$ symmetrizations in the $B\left(l_{1}^{n}\right)$ process, was to create almost Euclidean projections. This stage is unnecessary for the cube. Let us formulate the dual version of Lemma 4.2:

Lemma 5.2 Let $K=\frac{1}{\sqrt{n}} B\left(l_{\infty}^{n}\right)$, and let $E$ be a random subspace of dimension $\lambda n$. Then with high probability (greater than $1-e^{-c \lambda n}$ ),

$$
\operatorname{Proj}_{E}(K) \supset c_{1} \sqrt{\frac{1-\lambda}{-\log (1-\lambda)}} D_{n} \cap E
$$

Note that the constants $c_{1}, c_{2}, .$. in this section are not necessarily equal to the constants denoted by the same letters in the previous sections.

Lemma 5.3 Let $K=\frac{1}{\sqrt{n}} B\left(l_{\infty}^{n}\right)$, and let $E$ be a subspace of dimension $k$. Then:

$$
v . r .(K \cap E)>c_{2} \sqrt{\frac{k}{n}}
$$

where v.r. $(K \cap E)$ is calculated with respect to the natural Lebesgue measure in $E \subset \mathbb{R}^{n}$.

Proof: According to [V], for the cube of volume one, each section has volume at least one. Therefore:

$$
v . r .(K \cap E) \geq \frac{1}{\sqrt{n}\left(\operatorname{vol}\left(D_{n} \cap E\right)\right)^{1 / k}}>c_{2} \sqrt{\frac{k}{n}}
$$

Now we turn to describing the symmetrization process of $K$, and proving Proposition 5.1. Let $\left\{v_{1}, . ., v_{n}\right\} \in O(n)$ be a random orthonormal basis. Denote $\lambda_{i}=\frac{1}{\left(\log ^{(i)} n\right)^{2}}$, and $E_{i}=\operatorname{sp}\left\{v_{1}, . ., v_{\left\lfloor\lambda_{i} n\right\rfloor}\right\}$. An iteration transforms the body $K_{i-1}$ to $K_{i}$ (where $K_{0}=K$ ), in two steps:

- Symmetrize $K_{i-1}$ body with respect to any orthogonal basis in $E_{i}$, and obtain $K_{i}^{\prime}$.

- Use Theorem 2.7 for the body $K_{i}^{\prime} \cap E_{i}$. Symmetrize the entire body $K_{i}^{\prime}$ with respect to the vectors given by this theorem, to arrive at $K_{i}$.

Claim 5.4 For $i$ such that $\lambda_{i-1}<2 c_{3}$ (where $c_{3}=\frac{1}{\sqrt{2}} \min \left\{c_{1}, \bar{c}_{1} c_{2}\right\}$ ),

$$
c_{3} \sqrt{\frac{\lambda_{i}}{-\log \lambda_{i}}} D_{n} \subset K_{i}
$$


and not more than $(1+\bar{c})\left\lfloor\lambda_{i} n\right\rfloor \cdot 2 \log \frac{1}{\lambda_{i-1}}$ symmetrizations were used to construct $K_{i}$ from $K_{i-1}$.

Proof: By induction on $i$. For $i=0$ this is trivially true. For a general $i$, note that $K_{i}^{\prime}$ was constructed from $K$ using symmetrizations inside $E_{i}$. By Lemma 2.1 and Lemma 2.4,

$$
\operatorname{Proj}_{E_{i}^{\perp}}(K)=\operatorname{Proj}_{E_{i}^{\perp}}\left(K_{i}^{\prime}\right)=K_{i}^{\prime} \cap E_{i}^{\perp}
$$

Using Lemma 5.2, we get that:

$$
c_{1} \sqrt{\frac{\lambda_{i}}{-\log \lambda_{i}}} D_{n} \cap E_{i}^{\perp} \subset K_{i}^{\prime}
$$

Note that $K_{i-1} \subset D_{n}$. By the induction hypothesis,

$$
d_{B M}\left(K_{i}^{\prime}, D_{n}\right) \leq d\left(K_{i-1}, D_{n}\right) \leq \frac{\sqrt{-\log \lambda_{i-1}}}{c_{3} \sqrt{\lambda_{i-1}}}
$$

By Theorem 2.7, not more then $\bar{c}\left\lfloor\lambda_{i} n\right\rfloor \cdot \log 2 d_{B M}\left(K_{i}^{\prime}, D_{n}\right)$ symmetrizations were carried out to construct $K_{i}$ from $K_{i}^{\prime}$. Since $\lambda_{i-1}<2 c_{3}$, this number of symmetrizations is less than

$$
\bar{c}\left\lfloor\lambda_{i} n\right\rfloor \cdot 2 \log \frac{1}{\lambda_{i-1}}
$$

Because symmetrizations were carried out only inside $E_{i}$, then $\operatorname{vol}(K \cap$ $\left.E_{i}\right)=\operatorname{vol}\left(K_{i}^{\prime} \cap E_{i}\right)$. By Theorem 2.7 and Lemma 5.3, we can assure that

$$
\bar{c}_{1} c_{2} \sqrt{\lambda_{i}} D_{n} \cap E_{i} \subset K_{i}
$$

Since $K_{i}$ was obtained from $K_{i}^{\prime}$ using symmetrizations inside $E_{i}$, then (6) still holds for $K_{i}$. Combining (6) and (7), and the definition of $c_{3}$,

$$
c_{3} \sqrt{\frac{\lambda_{i}}{-\log \lambda_{i}}} D_{n} \subset K_{i}
$$

Now, take the minimal $t$ such that $\log ^{(t)} n>8(\bar{c}+1) / \varepsilon$ and also $\lambda_{t-1}<2 c_{3}$. To obtain $K_{t}$, we have used not more than

$$
\sum_{i<t}(1+\bar{c})\left\lfloor\lambda_{i} n\right\rfloor \cdot 2 \log \frac{1}{\lambda_{i-1}}<n \sum_{i<t} \frac{4(\bar{c}+1)}{\log ^{(i)} n}<\varepsilon n
$$

symmetrizations, and obtained a body of distance less than $O\left(e^{\frac{1}{\varepsilon} \log \frac{1}{\varepsilon}}\right)$ from the Euclidean ball. This dependence of $\varepsilon$ is worse than what was promised in Proposition 5.1. This is easy to fix. Instead of the 
prescribed iteration process, denote $E=s p\left\{v_{1}, . ., v_{\lfloor\varepsilon n\rfloor}\right\}$. By what we have just proved, there exist $4\lfloor\varepsilon n\rfloor$ symmetrizations carried out inside $E$, that transform the body $K$ into $K^{\prime}$, such that:

$$
c \sqrt{\varepsilon} D_{n} \cap E \subset c^{\prime} v \cdot r .(K \cap E) D_{n} \cap E \subset K^{\prime}
$$

where the first inclusion follows from Lemma 5.3. Since these $4\lfloor\varepsilon n\rfloor$ symmetrizations include symmetrizations with respect to an orthogonal basis of $E$, then by Lemma 5.2 and Lemma 2.4, we also have

$$
c_{1} \sqrt{\frac{\varepsilon}{-\log \varepsilon}} D_{n} \cap E^{\perp} \subset K^{\prime}
$$

Therefore, using $4\lfloor\varepsilon n\rfloor$ symmetrizations, we have obtained a body that contains a Euclidean ball of radius $O\left(\sqrt{\frac{\varepsilon}{-\log \varepsilon}}\right)$, which is an optimal dependence in $\varepsilon$, and Proposition 5.1 is proved.

By the discussion at the end of section 3, we have proved:

Theorem 5.5 Let $\varepsilon>0$ and let $K \subset \mathbb{R}^{n}$ be a convex body. Then there exist numbers $c_{1}(\varepsilon), c_{2}(\varepsilon)>0$, such that there exist $\lfloor(3+\varepsilon) n\rfloor$ Steiner symmetrizations that transform $K$ into a body $\tilde{K}$ that satisfies:

$$
c_{1}(\varepsilon) D_{n} \subset \frac{1}{v \cdot r .(K)} \tilde{K} \subset c_{2}(\varepsilon) D_{n}
$$

\section{Symmetrizing to an Ellipsoid}

We would like to investigate the smallest number of symmetrizations that transforms an arbitrary convex body $K \subset \mathbb{R}^{n}$ into an isomorphic Euclidean ball. Unfortunately, our technique leads to an exact answer of only a related question, where rather than approaching an isomorphic Euclidean ball, we obtain an isomorphic ellipsoid. Formally, for any convex body $K \subset \mathbb{R}^{n}$ define:

$$
S_{T}(K)=\inf \left\{d_{B M}\left(K_{T}, D_{n}\right) ; \exists H_{1}, . ., H_{n}, K_{T}=S_{H_{1}, . ., H_{T}}(K)\right\}
$$

the minimal distance to an ellipsoid, that can be achieved using $T$ symmetrizations. We are concerned with the following quantities, representing the asymptotic minimal number of symmetrizations:

$$
\begin{gathered}
S_{n}(c)=\min \left\{T ; \forall K \subset \mathbb{R}^{n} S_{T}(K) \leq c\right\} \\
\bar{S}(c)=\limsup _{n \rightarrow \infty} \frac{S_{n}(c)}{n}, \underline{S}(c)=\liminf _{n \rightarrow \infty} \frac{S_{n}(c)}{n}
\end{gathered}
$$

We do not know to prove that $\underline{S}(c)=\bar{S}(c)$, or that even these expressions are finite for values of $c$ close to 1 . However, we can prove the following: 
Theorem 6.1

$$
\lim _{c \rightarrow \infty} \underline{S}(c)=\lim _{c \rightarrow \infty} \bar{S}(c)=1
$$

The lower bound is easy to obtain:

Lemma 6.2 $\forall c>1 \underline{S}(c) \geq 1$

Proof: Take $K=B\left(l_{1}^{n}\right)$. Then for any subspace $H$ of dimension $k$ (cf. a dual of a theorem in [Ba] page 9$)$,

$$
d_{B M}\left(\operatorname{Proj}_{H}(K), D_{k}\right) \geq \sqrt{\frac{k}{2 \log n}}
$$

Apply any $T<n$ Steiner symmetrizations to $K$, with respect to the hyperplanes $H_{1}, . ., H_{T}$. Denote $H=\cap H_{i}$ and $K_{T}=S_{H_{1}, . ., H_{T}}(K)$. Then $\operatorname{dim}(H) \geq n-T$ and:

$$
\begin{gathered}
d_{B M}\left(K_{T}, D_{n}\right) \geq d_{B M}\left(\operatorname{Proj}_{H}\left(K_{T}\right), D_{n} \cap H\right)= \\
=d_{B M}\left(\operatorname{Proj}_{H}(K), D_{n} \cap H\right) \geq \sqrt{\frac{n-T}{2 \log n}}
\end{gathered}
$$

where the equality in the middle follows by Lemma 2.1. Plug in the definition of $S_{n}(c)$ :

$$
S_{n}(c) \geq n-2 c^{2} \log n \quad \Rightarrow \quad \underline{S}(c) \geq 1
$$

It remains to prove the upper bound. The proof makes an extensive use of the "quotient of subspace theorem", first proved in $[\mathrm{M}]$ for centrally symmetric bodies (see also $[\mathrm{P}]$, chapter 8 ). The extension to non-symmetric bodies appears in [MP2]. Let $K$ be a convex body. Its centroid or barycenter is the point $\int_{K} x d x \in K$. Denote $\bar{K}=\operatorname{conv}(K,-K)-$ the convex hull of $K$ and $-K$. Now we formulate a variant of the quotient of subspace theorem, which is easy to deduce from the references:

Theorem 6.3 Let $\varepsilon>0$ and let $K \subset \mathbb{R}^{n}$ be a convex body with 0 as a centroid, and with $\operatorname{vol}(K)=\operatorname{vol}\left(D_{n}\right)$. Then there exist $F \subset E \subset \mathbb{R}^{n}$ subspaces with $\operatorname{dim}(F)=\lceil(1-2 \varepsilon) n\rceil, \operatorname{dim}(E)>(1-\varepsilon) n$, and ellipsoid $\mathcal{E} \subset F$ such that:

$$
c_{1}(\varepsilon) \mathcal{E} \subset F \cap \operatorname{Proj}_{E}(K) \subset F \cap \operatorname{Proj}_{E}(\bar{K}) \subset c_{2}(\varepsilon) \mathcal{E}
$$


This theorem leads to the following lemma.

Lemma 6.4 Let $\varepsilon>0$ and let $K \subset \mathbb{R}^{n}$ be a convex body with 0 as its centroid. Then there exist $\lceil(1-\varepsilon) n\rceil$ Steiner symmetrizations, a positive number $\lambda$ and a subspace $F \subset \mathbb{R}^{n}$ with $\operatorname{dim}(F)=\lceil(1-2 \varepsilon) n\rceil$ such that the symmetrized body, $\tilde{K}$ satisfies:

$$
c_{1}(\varepsilon) \lambda D_{n} \cap F \subset \tilde{K} \cap F \subset \operatorname{Proj}_{F}(\tilde{K}) \subset c_{2}(\varepsilon) \lambda D_{n} \cap F
$$

Proof: According to Theorem 6.3, given $K \subset \mathbb{R}^{n}$ there exist some special subspaces $F \subset E \subset \mathbb{R}^{n}$, and a distinguished ellipsoid $\mathcal{E} \subset F$. Symmetrize $K$ with respect to any orthogonal basis in $E^{\perp}$, to obtain $\hat{K}$. By Lemma 2.4, $\hat{K} \cap E=\operatorname{Proj}_{E}(K)$, and by Theorem 6.3,

$$
c_{1}(\varepsilon) \mathcal{E} \subset \hat{K} \cap F \subset c_{2}(\varepsilon) \mathcal{E}
$$

According to Lemma 2.6, the ellipsoid $\mathcal{E}$ can be symmetrized to become $\lambda D_{\lceil(1-2 \varepsilon) n\rceil}$ using symmetrizations with respect to some orthogonal basis in $F$. Apply these symmetrizations to the entire body $\hat{K}$, to obtain $\tilde{K}$. A total of $\lceil(1-\varepsilon) n\rceil$ symmetrizations were carried. At this stage,

$$
c_{1}(\varepsilon) \lambda D_{n} \cap F \subset \tilde{K} \cap F \subset c_{2}(\varepsilon) \lambda D_{n} \cap F
$$

If we would apply exactly the same symmetrizations to the body $C=$ $\bar{K}$, then by Theorem 6.3 , we would obtain a centrally symmetric $\tilde{C}$ such that

$$
\operatorname{Proj}_{F}(\tilde{C})=\tilde{C} \cap F \subset c_{2}(\varepsilon) \lambda D_{n}
$$

where the equality follows by Lemma 2.5 , since $\tilde{C}$ is symmetric with respect to an orthogonal basis in $F$. Since $\tilde{K} \subset \tilde{C}$, we get that

$$
c_{1}(\varepsilon) \lambda D_{n} \cap F \subset \tilde{K} \cap F \subset \operatorname{Proj}_{F}(\tilde{K}) \subset c_{2}(\varepsilon) \lambda D_{n} \cap F
$$

Remark for Lemma 6.4: As can be easily deduced from the proof, a relatively small number of symmetrizations of an arbitrary convex body, may create very large sections which are uniformly isomorphic to some ellipsoid. Specifically, for any convex body $K \subset \mathbb{R}^{n}$ and $\varepsilon>0$ there exist a subspace $F$ of dimension $\lceil(1-2 \varepsilon) n\rceil$ and $\lfloor\varepsilon n\rfloor$ symmetrizations, such that for the symmmetrized body $\hat{K}$,

$$
d_{B M}\left(\hat{K} \cap F, D_{n} \cap F\right)<c(\varepsilon)
$$

Proof of Theorem 1.2: Let $K$ be a convex body. Assume that 0 is its centroid. Apply Lemma 6.4 to $K$. After $\lceil(1-\varepsilon)\rceil$ symmetrizations, we 
obtain $\hat{K}$ such that there exists a subspace $F$ with $\operatorname{dim}(F)=\lceil(1-2 \varepsilon) n$ and a number $\lambda_{1}$ with:

$$
c_{1}(\varepsilon) \lambda_{1} D_{n} \cap F \subset \hat{K} \cap F \subset \operatorname{Proj}_{F}(\hat{K}) \subset c_{2}(\varepsilon) \lambda_{1} D_{n} \cap F
$$

In addition, the symmetrizations from Lemma 6.4 include symmetrizations with respect to an orthogonal basis of $F$. By Lemma 2.4,

$$
\hat{K} \cap F^{\perp}=\operatorname{Proj}_{F^{\perp}} \hat{K}
$$

and further symmetrizations inside $F^{\perp}$ cannot hurt the validity of (8) or (9). By Proposition 5.5, there exist $4 \frac{\varepsilon n}{5}$ symmetrizations that transform $\hat{K} \cap F^{\perp}$ into an isomorphic Euclidean ball. Apply these symmetrizations to $\hat{K}$, to obtain $\tilde{K}$. Then:

$$
c_{1} \lambda_{2} D \cap F^{\perp} \subset \tilde{K} \cap F^{\perp}=\operatorname{Proj}_{F^{\perp}} \tilde{K} \subset c_{2} \lambda_{2} D \cap F^{\perp}
$$

Combining with $(8)$ we conclude that the unique ellipsoid $\mathcal{E}$ such that

$$
\begin{gathered}
\operatorname{Proj}_{F} \mathcal{E}=F \cap \mathcal{E}=\lambda_{1} D_{n} \cap F \\
\operatorname{Proj}_{F^{\perp}} \mathcal{E}=F^{\perp} \cap \mathcal{E}=\lambda_{2} D_{n} \cap F^{\perp}
\end{gathered}
$$

satisfies

$$
c_{1}(\varepsilon) \mathcal{E} \subset \tilde{K} \subset c_{2}(\varepsilon) \mathcal{E}
$$

and the proposition is proved, for bodies with 0 as a centroid.

It remains to get rid of the assumption that 0 is the centroid of $K$. Write $b(K)=\frac{1}{v o l(K)} \int_{K} x d x$ the centroid of $K$. We have described a short symmetrization process for the body $K-b(K)$, resulting with the body $\tilde{K}$. We claim that when these symmetrizations are applied to $K$, the resulting body is again, exactly $\tilde{K}$. Indeed, note that:

$$
b\left(S_{H}(K)\right)=\operatorname{Proj}_{H}(b(K))
$$

Since we symmetrize with respect to an orthonormal basis of $F$, and also with respect to an orthonormal basis of $F^{\perp}$, the resulting body has 0 as a centroid. Therefore, the symmetrizations of $K-b(K)$ and $K$ have the same centroid, and since apriori they differ only by a translation they must be identical. Thus, Proposition 1.2 is finally proved.

Plugging in the estimates from "quotient of subspace" theorem, we get that the distance to an ellipsoid in Proposition 1.2 is of the order of $O\left(\varepsilon^{-1} \log \varepsilon^{-1}\right)$. It is clear that Theorem 6.1 follows from Proposition 1.2 and Lemma 6.2. The deduction of Theorem 1.3 is easy; Given an arbitrary convex body, apply the symmetrizations of Proposition 1.2 to the body. Then, symmetrize the resulting "isomorphic ellipsoid" into an isomorphic Euclidean ball, according to Lemma 2.6, using $n$ additional Steiner symmetrizations. To conclude, we obtained an isomorphic Euclidean ball from an arbitrary convex body, using a total of $\lfloor(2+\varepsilon) n\rfloor$ symmetrizations. 


\section{References}

[Ba] K.M. Ball, An Elementary Introduction to Modern Convex Geometry, appeared in Flavors of Geometry, Ed. S. Levy, MSRI vol. 31, Cambridge Univ. Press (1997).

[B1] H. Brunn, Über Ovale and Eiflächen, Dissertation, München (1887).

[B2] H. Brunn, Referat Über eine Arbeit: Exacte Grundlageg für einer Theorie der Ovale, (S.B. Bayer, ed.) Akad. Wiss. (1894) 93-111.

$[\mathrm{BF}]$ T. Bonnesen, W. Fenchel, Theorie der konvexen Körper. Springer, Berlin (1934); English transl. Theory of convex bodies, BCS associates (1987).

[BN] S.G. Bobkov, F.L. Nazarov, On convex bodies nad log-concave probability measures with unconditional bodies. To appear on Geometric aspects of functional analysis, Springer LNM (2002).

[BLM] J. Bourgain, J. Lindenstrauss, V.D. Milman, Estimates related to Steiner symmetrizations. Geometric aspects of functional analysis (1987-88), Lecture Notes in Math., vol. 1376, Springer Berlin, (1989) 264-273.

[BZ] Yu.D. Burago, V.A. Zalgaller, Geometric inequalities. Springer Series in Soviet Mathematics. Springer-Verlag, Berlin (1988).

[CS] C. Caratheodory, E. Study, Zwei Beweise des Satzes, daß der Kreis unter allen Figuren gleichen Umfanges den größten Inhaly hat, Math. Annalen 68 (1909) 133-144 (in German).

[GG] A. Yu. Garnaev, E.D. Gluskin, The widths of a Euclidean ball, Dokl. Akad. Nauk SSSR 277, no. 5, (1984) 1048-1052; English transl. in Soviet Math. Dokl. 30 (1984).

[GM] A.A. Giannopoulos, V.D. Milman, Euclidean structures in finite dimensional normed spaces. Handbook of the Geometry of Banach spaces, W. B. Johnson, J. Lindenstrauss eds., Elsevier Science, Vol. 1 (2001), 707-779.

$[\mathrm{H}]$ H. Hadwiger, Einfache Herleitung der isoperimetrischen Ungleichung für abgeschlossene Punktmengen, Math. Ann. 124 (1952), 158-160.

[K] B. Klartag, 5n Minkowski symmetrizations suffice to arrive at an approximate Euclidean ball, to appear in Ann. Math.

[Ka] B.S. Kashin, Sections of some finite-dimensional sets and classes of smooth functions, Izv. Akad. Nauk. SSSR, Ser. Mat. 41 (1977) 334-351; English transl. in Math. USSR Izv. 11 (1977).

[Mac] A.M. Macbeath, An extremal property of the hypersphere. Proc. Camb. Phil. Soc. 47 (1951), 245-247. 
[Man] P. Mani-Levitska, Random Steiner symmetrizations, Studia Sci. Math. Hung. 21, (1986) 373-378.

[MeP] M. Meyer, A. Pajor, On the Blaschke-Santalo inequaqlity, Arch. Math. Birkhäuser verlag, vol. 55 (1990) 82-93.

[M] V.D. Milman, Almost Euclidean qoutient spaces of subspaces of a finite dimensional normed space, Proc. Amer. Math. Soc. 94 (1985) 445-449.

[MP1] V.D. Milman, A. Pajor, Isotropic position and inertia ellipsoids and zonoids of the unit ball of a normed $n$-dimensional space. Geometric aspects of functional analysis (1987-88), Lecture Notes in Math., vol. 1376, Springer Berlin, (1989) 64-104.

[MP2] V.D. Milman, A. Pajor, Entropy and Asymptotic Geometry of Non-Symmetric Convex Bodies, Advances in Math., vol. 152, no. 2 (2000) 314-335.

[MS] V.D. Milman, G. Schechtman, Asymptotic theory of finitedimensional normed spaces. Lecture Notes in Math., vol. 1200, Springer-Verlag, Berlin (1986).

[P] G. Pisier, The volume of convex bodies and Banach space geometry, Cambridge Tracts in Mathematics, Cambridge univ. Press, vol. 94 (1997).

[Sch] R. Schneider, Convex bodies: The Brunn-Minkowski theory, Encyclopedia of Mathematics and its Applications, Cambridge Univ. Press, vol. 44 (1993).

[S] J. Steiner, Einfacher Beweis der isoperimetrischen Hauptsätze, Crelle J. Reine Angew. Math 18 (1838) 281-296; Ges. Werke 2 (Berlin, 1882), 77-91 (in German).

[V] J. D. Vaaler, A geometric inequality with applications to linear forms, Pacific Jour. of Math., vol. 83, no. 2 (1979) 543-553. 